\title{
Fast Tangentially Viewed Soft X-Ray Imaging System Based on Image Intensifier with Microchannel Plate Detector on QUEST
}

\author{
Canbin HUANG, Kazuaki HANADA ${ }^{1)}$, Kengoh KURODA ${ }^{1)}$, Shinichro KOJIMA ${ }^{1)}$, \\ Hiroaki FUJIYOSHI $^{1)}$, Hiroki MIURA ${ }^{1)}$, Tomoki YAMADA ${ }^{1)}$, Hiroshi IDEI ${ }^{1)}$, \\ Makoto HASEGAWA ${ }^{1)}$ and Takumi ONCHI ${ }^{1)}$ \\ Interdisciplinary Graduate School of Engineering and Sciences, Kyushu University, Kasuga 812-8580, Japan \\ ${ }^{1)}$ Research Institute for Applied Mechanics, Kyushu University, Kasuga 812-8580, Japan
}

(Received 15 April 2019 / Accepted 11 June 2019)

\begin{abstract}
A two-dimensional (2D) soft X-ray (SXR) imaging system for obtaining tangential views of plasmaproduced SXR has been installed on the QUEST tokamak. The system comprises a chevron micro-channel plate (MCP) and a phosphor screen, which together serve as an SXR detector and image intensifier, along with a high-speed video camera for capturing and storing image data. The system can be used in imaging mode to obtain high spatial- and time-resolution images or in photon counting mode at an ultra-high framing rate of $100 \mathrm{kHz}$ to obtain SXR energy spectra. Here, we propose a method of in situ energy calibration based on the transmission characteristics of a $25-\mu \mathrm{m}$ Be filter. The proposed method was used to obtain SXR spectra in the energy range $1.5-3.5 \mathrm{keV}$ with a core electron temperature in agreement with electron temperatures measured by the Thomson scattering method. Furthermore, oscillations based on the modification of plasma parameters and rapid increases in impurity radiation were detected in imaging mode. This dual-mode-compatible method for obtaining 2D SXR diagnostics is useful for the study of high temperature plasmas.
\end{abstract}

(C) 2019 The Japan Society of Plasma Science and Nuclear Fusion Research

Keywords: soft X-ray imaging, MCP, photon counting

DOI: $10.1585 /$ pfr.14.1402128

\section{Introduction}

Soft X-ray (SXR) diagnostics have been commonly used to study a number of high-temperature plasma phenomena, including magnetohydrodynamic activity [1], plasma shaping control [2], and impurity contamination [3]. One-dimensional scintillator-based arrays [4] and diode-based arrays [5, 6] have been widely used in the tomographic two-dimensional (2D) reconstruction of X-ray emission images; however, the spatial resolution of SXR arrays is limited. Another problem with SXR measurement is the difficulty of identifying the physical meanings of signals. The characteristics of SXR emissions depend on the plasma temperature, density, and impurity content and can be used to capture variations in the plasma, but it remains difficult to clarify which plasma parameters have varied.

Direct 2D SXR imaging with high spatial and time resolutions has recently been made possible through the use of charge coupled device (CCD) cameras sensitive to SXR [7] or image intensifiers comprising a microchannel plate (MCP) detector and a phosphor screen coupled to a high-speed video camera $[8,9]$. The image intensifier of an MCP detector can measure SXR signals with higher gain [10] than SXR cameras, enabling their use in lowdensity plasmas. In this study, we attempted to exploit

author'se-mail: huang@triam.kyushu-u.ac.jp these diagnostic abilities to propose a mode-compatible application for MCP detectors. The time resolution of an MCP-based diagnostic system is limited by that of the high-speed video camera, which generally has the capability of taking data every 10 us. The camera can be used in one of two modes-photon counting or imaging modewhich are selected by setting the time resolution of the high-speed camera. The diagnostics of photon counting mode can be used to obtain an SXR energy spectrum corresponding to an accurate electron temperature; in imaging mode, the high-speed camera output can be used to produce a time-integration SXR signal as a very high-spatialresolution 2D SXR image that can be used to measure rapid variations in the plasma.

In this study, we looked at how mode selection could be applied in measuring the characteristics of the hightemperature plasmas produced by QUEST and resolved a number of difficulties in applying the respective modes. Typical examples of our results are discussed in this paper to demonstrate how the compatible use of modes can extend the potential of SXR measurement to obtain further understanding of the properties of plasma.

\section{Experimental Setup}

The Q-shu University Experiment with Steady-state Spherical Tokamak (QUEST) is a medium-size spherical 


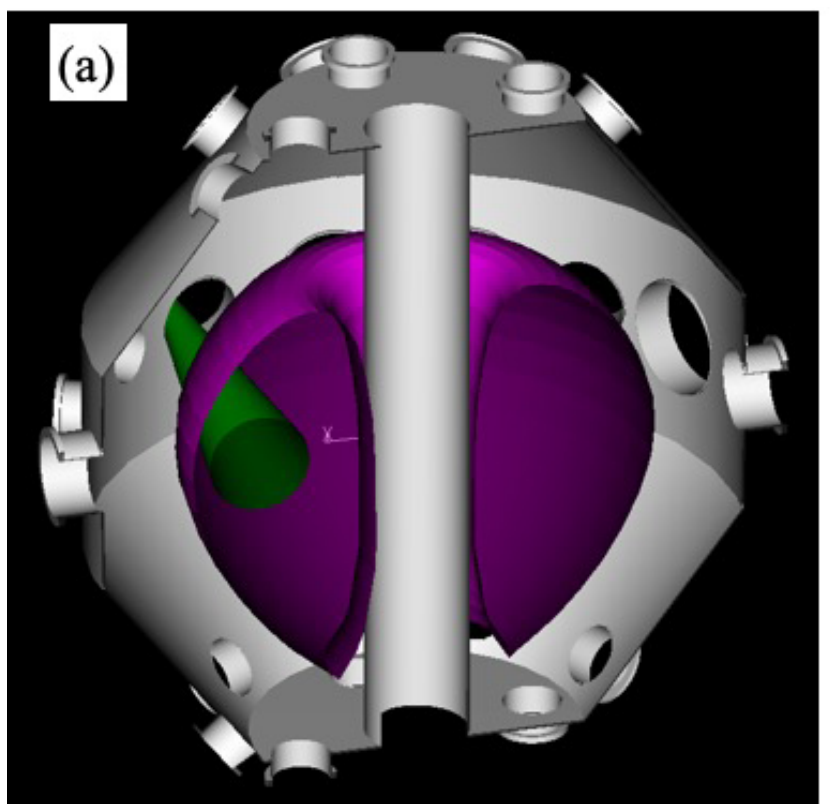

(b)

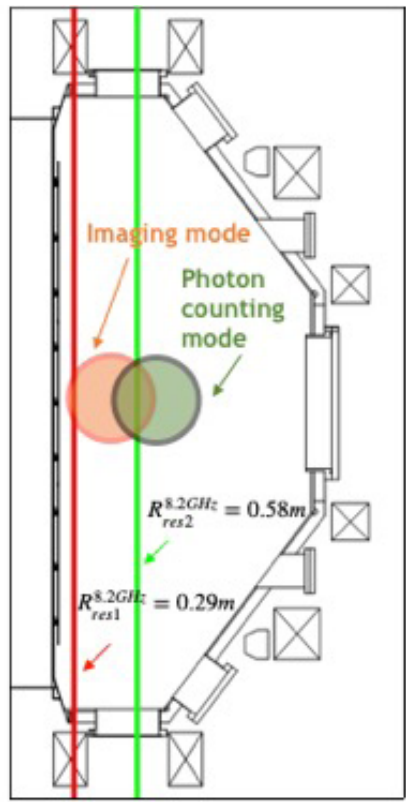

Fig. 1 Schematic of FOV of the tangential SXR imaging system on QUEST. (a) 3-D view; (b) poloidal section with movable FOV (red color circle in imaging mode and green color circle in photon counting mode). Fundamental and second harmonic resonance layers of $8.2 \mathrm{GHz} \mathrm{ECW}$ at $B_{t}=0.13 \mathrm{~T}$ are denoted by red and green lines.

tokamak (ST) device [11] with major and minor radii of $R=0.64 \mathrm{~m}$ and $a=0.4 \mathrm{~m}$, respectively, and major limiter radii of 0.22 and $1.32 \mathrm{~m}$ on the mid-plane. All of the QUEST discharges discussed in this paper were produced in an inboard limiter (IL) configuration [12]. Two electron cyclotron wave (ECW) systems operating at 8.2 and $28 \mathrm{GHz}$ are used for current start-up and current driving, respectively [13-15]. A center solenoid (CS) coil is used for Ohmic heating [16].

The SXR imaging system was installed at a tangential port on QUEST. The system watches the core region of QUEST plasma tangentially and the ECW harmonic resonance layers at the typical toroidal magnetic field. When $B_{t}$ is fixed at $0.13 \mathrm{~T}$ at the center of the vacuum vessel ( $R=0.64 \mathrm{~m}$ ), the fundamental and second harmonic resonance layers of $8.2 \mathrm{GHz} \mathrm{ECW}$ are located at $R_{\text {res } 1}=0.29 \mathrm{~m}$ and $R_{\text {res } 2}=0.58 \mathrm{~m}$, respectively. Figure 1 (a) shows 3-D field of view (FOV) of the system. The viewing area is a range of 36-cm circle at the poloidal section of QUEST, which is changeable at radial direction by moving the rear end of the system. Figure 1 (b) shows the typical FOV at imaging mode $(R=0.26-0.62 \mathrm{~m})$ and photon counting mode $(R=0.48-0.84 \mathrm{~m})$ in this paper.

Figure 2 shows a schematic of the QUEST 2D SXR diagnostics. The incoming SXR flux can be adjusted by changing the size of the pinhole (between 1, 2, 4, and $6 \mathrm{~mm}$ ) using a slider. A 30-mm-thick tungsten mask with a $\mathrm{V}$-shaped hole is mounted at the front of the pinhole plate as a shield against hard X-rays. A 5- $\mu$ m-thick Al filter mounted on a shutter is used to prevent visible and ultraviolet (UV) photons from entering the imaging system. The second, 25- $\mu \mathrm{m}$-thick Be filter mounted on the plate can be used to cover half of the four pinholes. The two filters can be used independently for detection of SXR emissions above $600 \mathrm{eV}$ or together for energy calibration in photon counting mode. The details of these methods are described in Sec. 3.

The core component of the SXR imaging system is a chevron detector assembly (PHOTONIS USA, INC) comprising a two-stage MCP assembly and a fiberoptic P46 phosphor screen mounted to a $6.0^{\prime \prime}$ vacuum flange. The MCP serves as an SXR detector and image intensifier with an SXR detection efficiency of $5-15 \%$ in the energy range $0.02-10 \mathrm{keV}$ [17]. SXR photons incident on the MCP produce photoelectrons that induce an MCP electron cascade via a secondary emission process. These secondary electrons are transformed by the phosphor screen into visible images that are recorded by a fast camera (PHOTRON, INC. Model FASTCAM SA-X). The specified gain of the MCP is up to $1 \times 10^{7}$ at an operational voltage of $1.8 \mathrm{kV}$; gains of this magnitude have been shown to be useful in monitoring the relatively low-density and -temperature QUEST plasma. The gain performance of the $\mathrm{MCP} /$ phosphor assembly was tested experimentally using MCP and phosphor voltages of 1.5 and $3.0 \mathrm{kV}$, respectively, as references. As shown in Fig. 3, increasing the MCP voltage from 1.5 to $2.0 \mathrm{kV}$ increased the image intensity by a factor of 11 ; by contrast, increasing the phosphor voltage from 3.0 to $5.0 \mathrm{kV}$ increased the intensity by only a factor of 2.5. According to the gain model in [10], the gain of an MCP has an exponential dependence on the MCP voltage. As shown in Fig. 3 (a), the gain begins to exponentially increase at MCP voltages above $1.8 \mathrm{kV}$ as a result of current saturation in some of the MCP channels. 


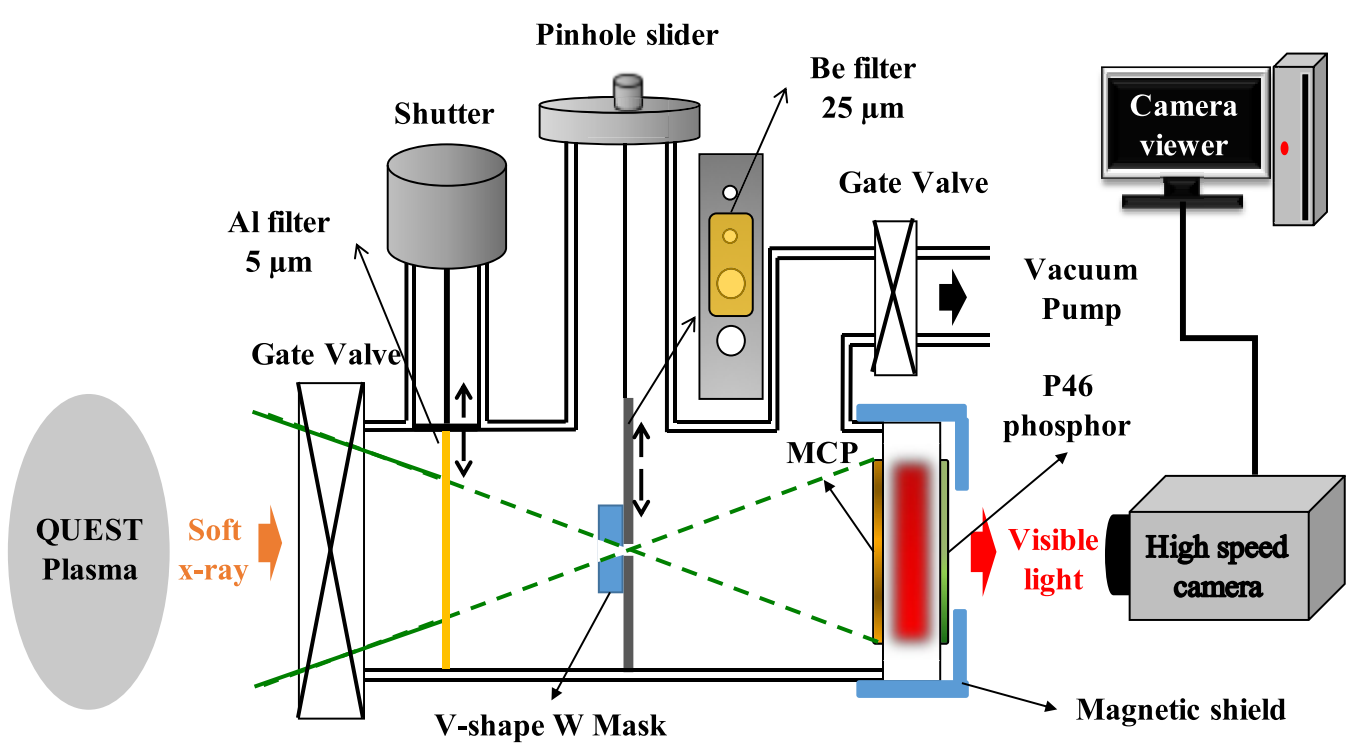

Fig. 2 Schematic of the SXR imaging system on QUEST. A 25- $\mu$ m-thick Be filter mounted on 1 and 6-mm pinhole plates is displayed; another set of 2- and 4-mm pinhole plates is also available.
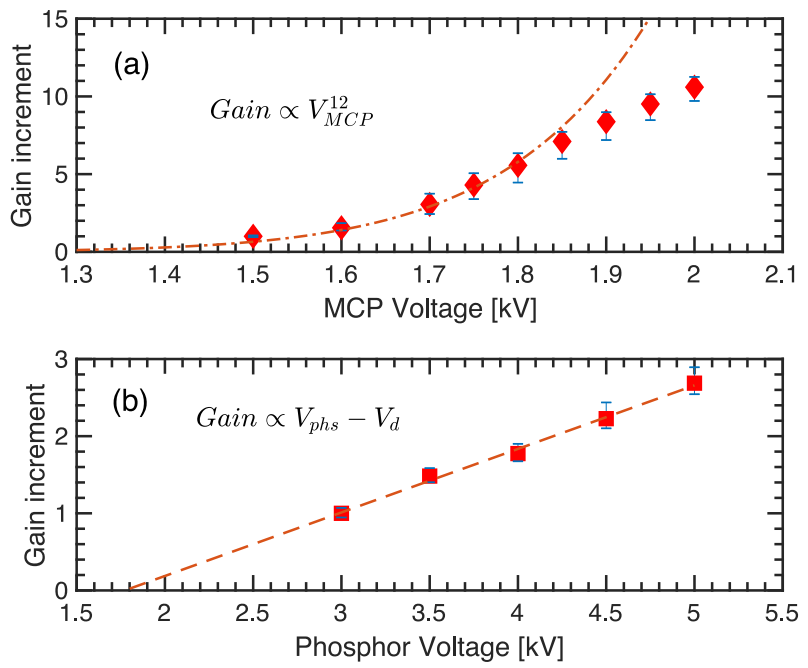

Fig. 3 Variation of image intensity during bench test voltage scan of: (a) MCP and (b) phosphor screen. The upper and lower limit data are obtained from the intensity variation over 200 frames. Exponential and linear fitting were applied to the MCP and phosphor data, respectively.

In addition to high gain, the MCP has ultra-high time $(<100 \mathrm{ps})$ and spatial resolutions $(12-\mu \mathrm{m}$-diameter channels with $15-\mu \mathrm{m}$ center-to-center spacing); the decay time constant of the P46 phosphor screen is also very short (about 70 - $80 \mathrm{~ns}$ ) [18], which allows the system to measure SXR emissivity with high spatial and time resolutions in imaging mode. The MCP is also capable of single-photon detection and can operate in photon counting mode using the $100 \mathrm{kHz}$ maximum framing rate available to the fast camera.
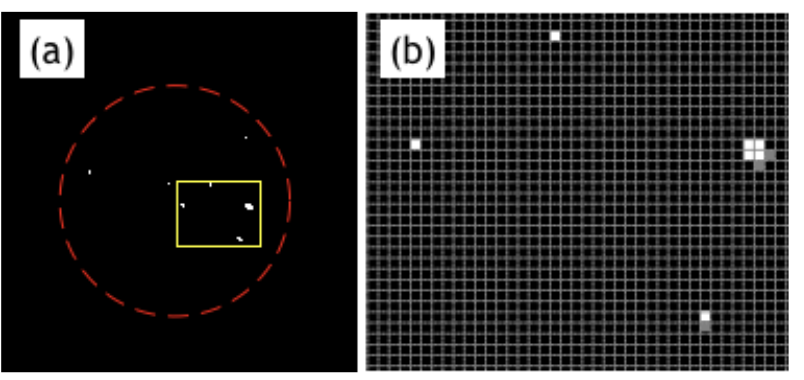

Fig. 4 (a) Photon counting frame. The red dash circle denotes the imaging area of the MCP assembly. (b) Pixel information of yellow rectangular region in left image. Some photons occupy more than one pixel.

\section{Photon Counting Mode Applica- tion}

The MCP detector can obtain QUEST plasma SXR spectra in photon counting mode. Figure 4 show a typical photon counting image taken at the fast camera framing rate of $100 \mathrm{kHz}$. X-ray photons are detected at a rate of seven counts per $256 \times 256$ pixels in each frame. This photon flux is low enough to achieve a complete photon counting mode in which no double X-ray photons are detected in a given pixel. It is seen that some photons occupy two or more pixels in a manner similar to the split events produced in SXR CCD camera images [7] as a result of spreading of the finitely sized electron clouds produced by individual X-ray photons into neighboring pixels. To determine the energy of such X-ray photons accurately, the photon intensity of electron spreading to two or more pixels is integrated. 
The energy of an SXR spectrum is typically calibrated against that of an X-ray source [7,19]. However, the gain of the SXR imaging system varies with the applied MCP and phosphor voltages, making conventional calibration difficult. Here, we propose a new in situ calibration method based on the transmission curve of a Be filter. Over the MCP and phosphor voltage ranges discussed in the preceding section, we performed photon counting over two consecutive shots with the same plasma parameters but different filters—an Al filter and an "Al+Be" filter-applied. The ratio of SXR photons detected using the respective filters is given by

$$
\begin{aligned}
\Re(\text { In }) & =\frac{N_{A l+B e}}{N_{A l}}=\frac{\Psi_{p} \cdot\left(T_{A l} \cdot T_{B e}\right) \cdot T_{M C P}}{\Psi_{p} \cdot T_{A l} \cdot T_{M C P}} \\
& =T_{B e}(h v),
\end{aligned}
$$

where $\Psi_{p}$ is the SXR photon flux from the plasma, $N_{A l+B e}$ and $N_{A l}$ are the photon numbers obtained by the MCP detector with the "Al+Be" and $\mathrm{Al}$ filters, respectively, $T_{A l}$ and $T_{B e}$ are the transmission rates of the $\mathrm{Al}$ and Be filters, respectively, $T_{M C P}$ is the quantum detection efficiency of the $\mathrm{MCP}$, In is the intensity of an image produced by an individual incident photon, and $h v$ is the SXR photon energy.

The energy calibration (i.e. the relationship between $I n$ and $h v$ ) is determined in the following process. According to [20], the Be filter transmission can be approximated using the following equation

$$
T_{B e}=\exp \left(-\frac{E_{0}^{3}}{[h v]^{3}}\right),
$$

where $E_{0}=(\log 2)^{1 / 3} E_{c, 50 \%}$, and the $50 \%$ cutoff energy of $25-\mu \mathrm{m}$ Be filter is $E_{c, 50 \%}=1588.4 \mathrm{eV}$. Combining Eq. (2) with $\Re(I n)=T_{B e}(h v)$, photon energy can be expressed as

$$
h v=\frac{E_{0}}{\left[-\log \left(T_{B e}\right)\right]^{1 / 3}}=\frac{E_{0}}{[-\log (\Re)]^{1 / 3}} .
$$

Finally, the energy calibration function is determined by a linear fitting of $I n$ and $h v$ data using least squares method.

The data and results of the energy calibration are shown in Fig. 5. Figure 5 (a) shows the photon counting data of $N_{A l}$ and $N_{A l+B e}$, and Fig. 5 (b) shows the ratio data $\Re$ and calibrated $T_{B e}$ curve. It should be noted that for In $<25$, the ratio $\Re$ is about $0.1 \sim 0.2$, higher than what we expect from the Be transmission curve. The data in low energy part is curious and remove from the fitting. The several candidates are considered, but we could not have any conclusion yet. Therefore, this part is removed in the energy calibration. For $I n \geq 60$, the ratio $\Re$ reaches 0.8 and starts to fluctuate around 1.0. This is because $T_{B e}$ is close to one, so $N_{A l+B e}$ is close to $N_{A l}$. The error bar in $\Re$ is larger because the photon number is smaller with large error. We used 35 points in $25 \leq$ In $<60$ for energy calibration, which gives the energy calibration function:

$$
h v=24 \times I n+590[\mathrm{eV}] .
$$
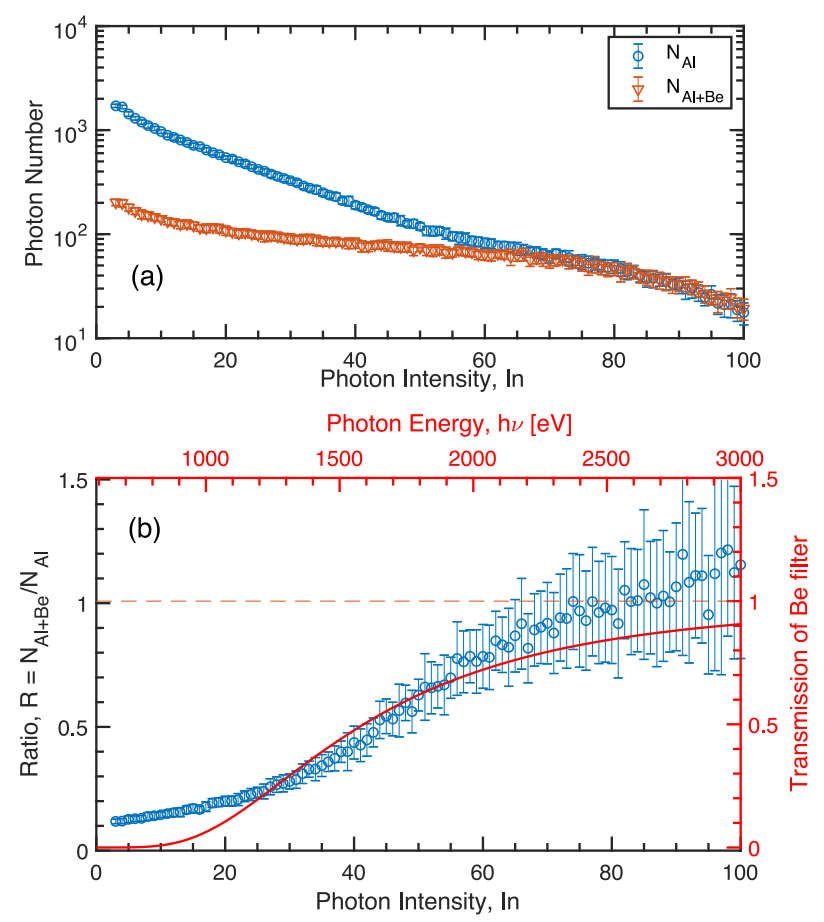

Fig. 5 (a) Photon counting number with $\mathrm{Al}$ filter and "Al+Be" filter. (b) Left-hand and lower axes: ratios of photon numbers with "Al+Be" and $\mathrm{Al}$ filters to photon intensity, In. $\Re=N_{A l+B e} / N_{A l}$. Data obtained from 260,000 frames in photon counting mode are divided into 26 groups. The error bars denote the standard deviation of data. Righthand and top axes: transmission curve of $25-\mu \mathrm{m}$ Be filter as a function of photon energy, $T_{B e}(h v)$.

Here the Be filter transmission of the intercept $590 \mathrm{eV}$ is $T_{B e}<1 \times 10^{-5}$, close to 0 . The coefficients with $95 \%$ confidence bound in the fitting is $24 \pm 2$, and the intercept with $95 \%$ confidence bound in the fitting is $590 \pm 90$.

Figure 6 shows the SXR spectra in the energy range $1.5-3.5 \mathrm{keV}$ following calibration and correction. This energy range is free of the characteristic radiation lines of metal and light impurities that are produced in other tokamak devices [19]. Here, we compare the energy spectra produced by the left and right halves of the viewing area. In the shots, which were conducted using a limiter configuration, plasma currents of $6 \mathrm{kA}$ were sustained noninductively by an $8.2 \mathrm{GHz}, 40 \mathrm{~kW}$ ECW power supply with the value of $B_{t}$ fixed at $0.13 \mathrm{~T}$. The calculated bulk plasma temperature (lower component) for each half is about 120 $140 \mathrm{eV}$, a result consistent with Thomson scattering measurements for an inboard limiter configuration. It is seen that the SXR spectra have a temperature component that is much higher than the bulk temperature, clearly indicating the presence of higher-energy components in the RF current-driven plasma that might play an essential role in non-inductive current driving. Although this information is potentially useful in understanding how plasma current can be driven by RF, the associated physical analysis exceeds 

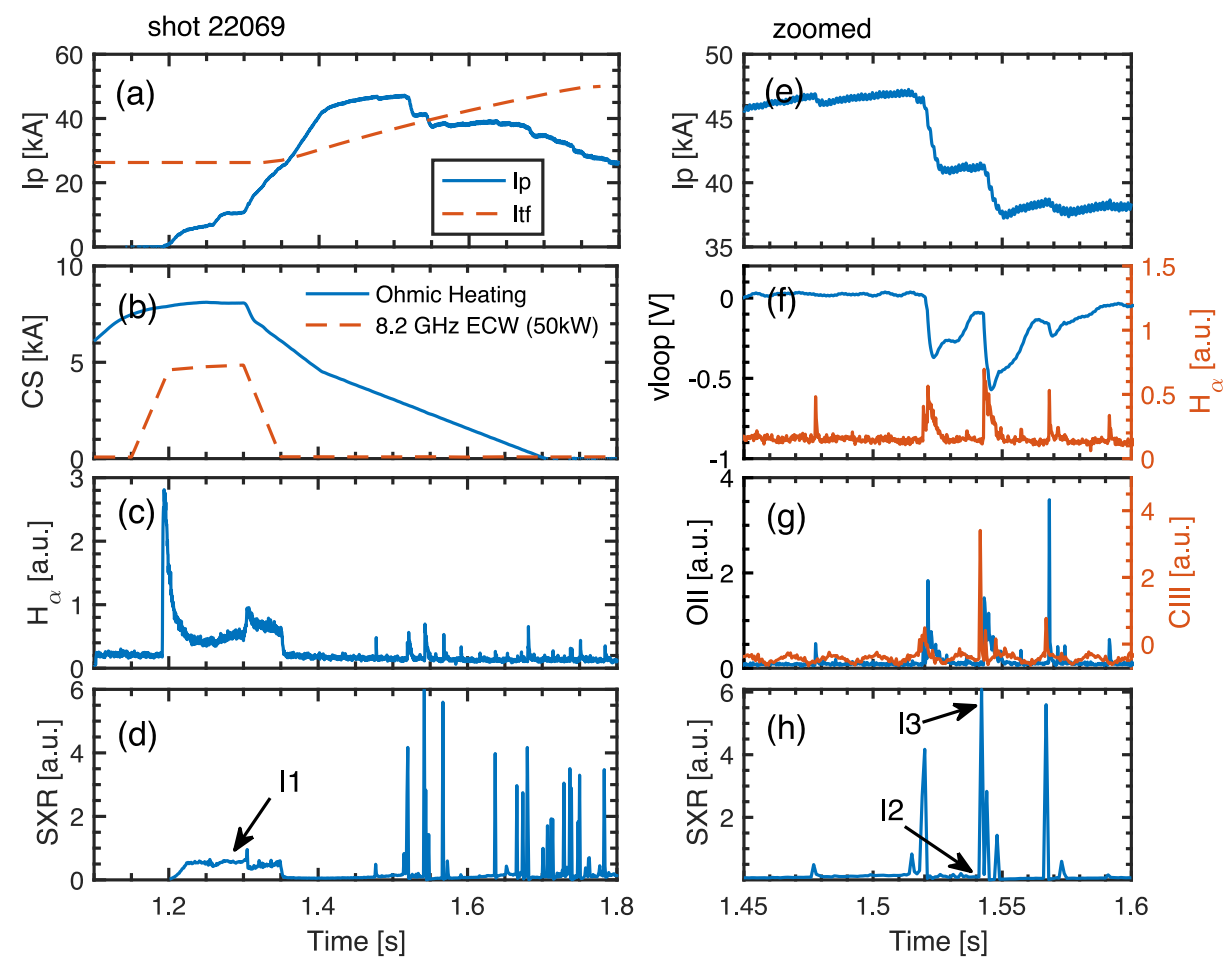

Fig. 7 Waveforms of: (a) plasma current, $I_{p}$, and toroidal field coil current, $I_{T F}$; (b) Ohmic heating by CS coil and ECW heating power; (c) hydrogen Balmer line radiation, $H_{\alpha}$; and (d) SXR intensity. (e - h) Zoomed waveforms of: (e) $I_{p}$; (f) loop voltage and $H_{\alpha}$; (g) oxygen-2 line radiation OII and carbon-3 line radiation CIII; and (h) SXR intensity. I1-I3 denote the timing of SXR images in Fig. 8.

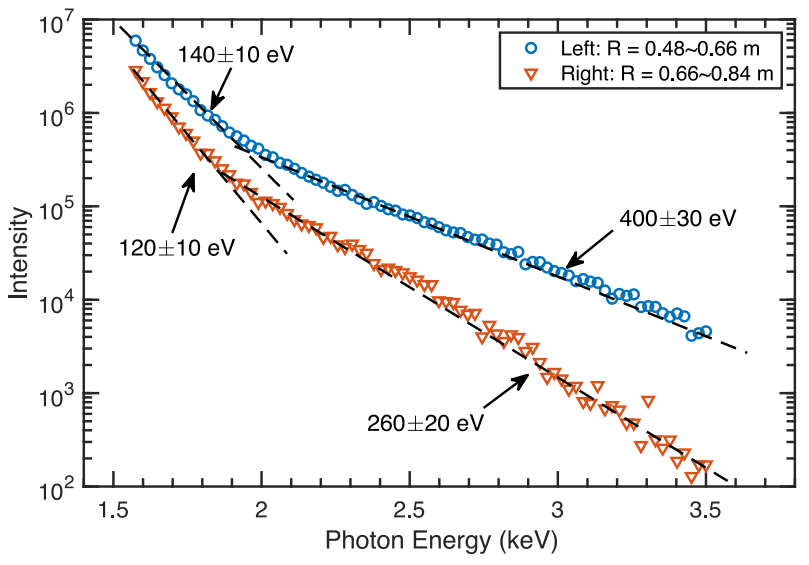

Fig. 6 SXR energy spectra corrected using the quantum efficiency of MCP detector and filter transmission. The imaging area of the MCP assembly is divided into a left half $(R=0.48-0.66 \mathrm{~m})$ and a right half $(R=0.66$ $0.84 \mathrm{~m})$. Core plasma temperatures of 120 and $140 \mathrm{eV}$ is derived from the fitting. The error bar of temperature is estimated using the $95 \%$ confidence bound in energy calibration.

the scope of this paper. Because the second harmonic resonance layer lies in left half (high field side), as shown in Fig. 1 (b), the measured bulk electron temperature in this half is higher than that in the right half (low field side).

\section{Imaging Mode Application 4.1 Enhancing SXR impurity radiation via slide-away electrons}

Non-inductive current ramp-up has previously been obtained in QUEST through the use of ECW [13, 14], and the generation and confinement of energetic electrons has been shown to play an important role in driving plasma current [12]. Normally, the presence of energetic electrons is indicated by the hard X-rays produced when the electrons hit a wall. Here, we propose an SXR imaging systembased detection method that measures the effects produced by slide-away electrons.

We used an $8.2 \mathrm{GHz}$ electron cyclotron current drive (ECCD) to inductively drive a $10-\mathrm{kA}$ plasma current during the period $t=1.2-1.35 \mathrm{~s}$ (Fig. 7), with $B_{t}$ increased from $0.13 \mathrm{~T}$ at $1.35 \mathrm{~s}$ to $0.26 \mathrm{~T}$ at $1.8 \mathrm{~s}$. Figures $7(\mathrm{~d})$ and 8 (a) show imaging system measurements of the SXR radiation from the hydrogen plasma at around $t=1.2-1.35 \mathrm{~s}$. The SXR intensity signals in Figs. 7 (d) and (h) were calculated by summing the SXR image intensities in each frame. After inductively increasing to about $50 \mathrm{kA}$, the plasma current began to decrease as the ECW power and Ohmic heating were turned off. After the ECW power was stopped at $1.3 \mathrm{~s}$, the CS coil current was decreased to drive and decelerate the plasma current, which began to decrease from $1.5 \mathrm{~s}$. As shown in Figs. $7(\mathrm{e}-\mathrm{h})$, the drop in plasma current created an enhancement of the loop voltage applied 

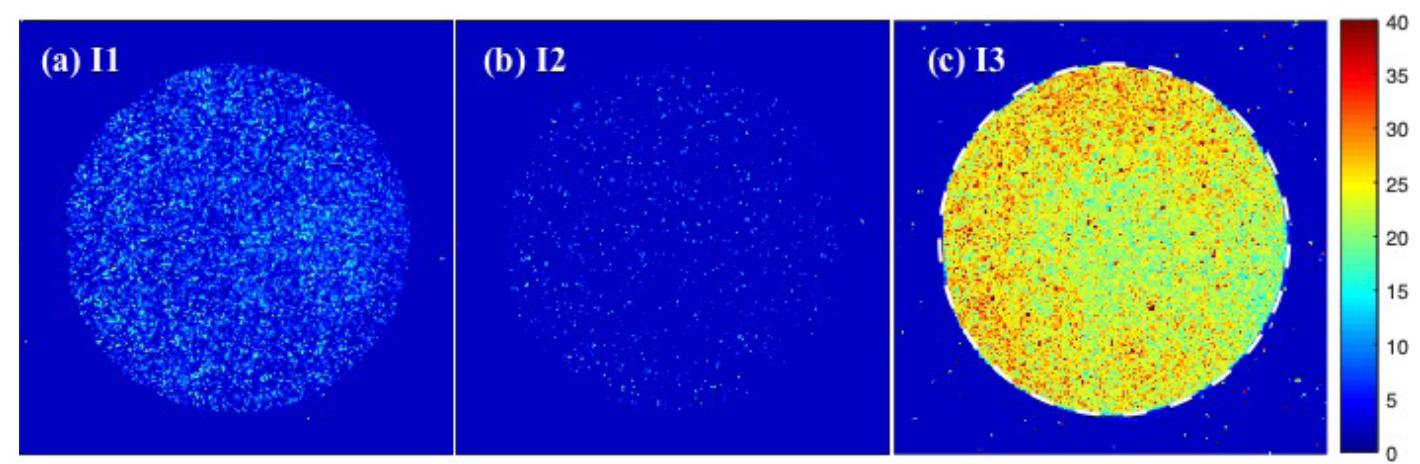

Fig. 8 Images of SXR emissivity (false color) in shot 22069: framing rate is $1 \mathrm{kHz}$. The timing of (a) I1, (b) I2, and (c) I3 images are shown in Figs. 7 (d) and (h). The white dashed circle in (c) denotes the imaging area of the MCP assembly. Spots outside of the MCP area are caused by penetrating HX emission. The range of color map is $[0,40]$. The $8.2 \mathrm{GHz}$ resonance layer is outside (at the left side) of the observation window.
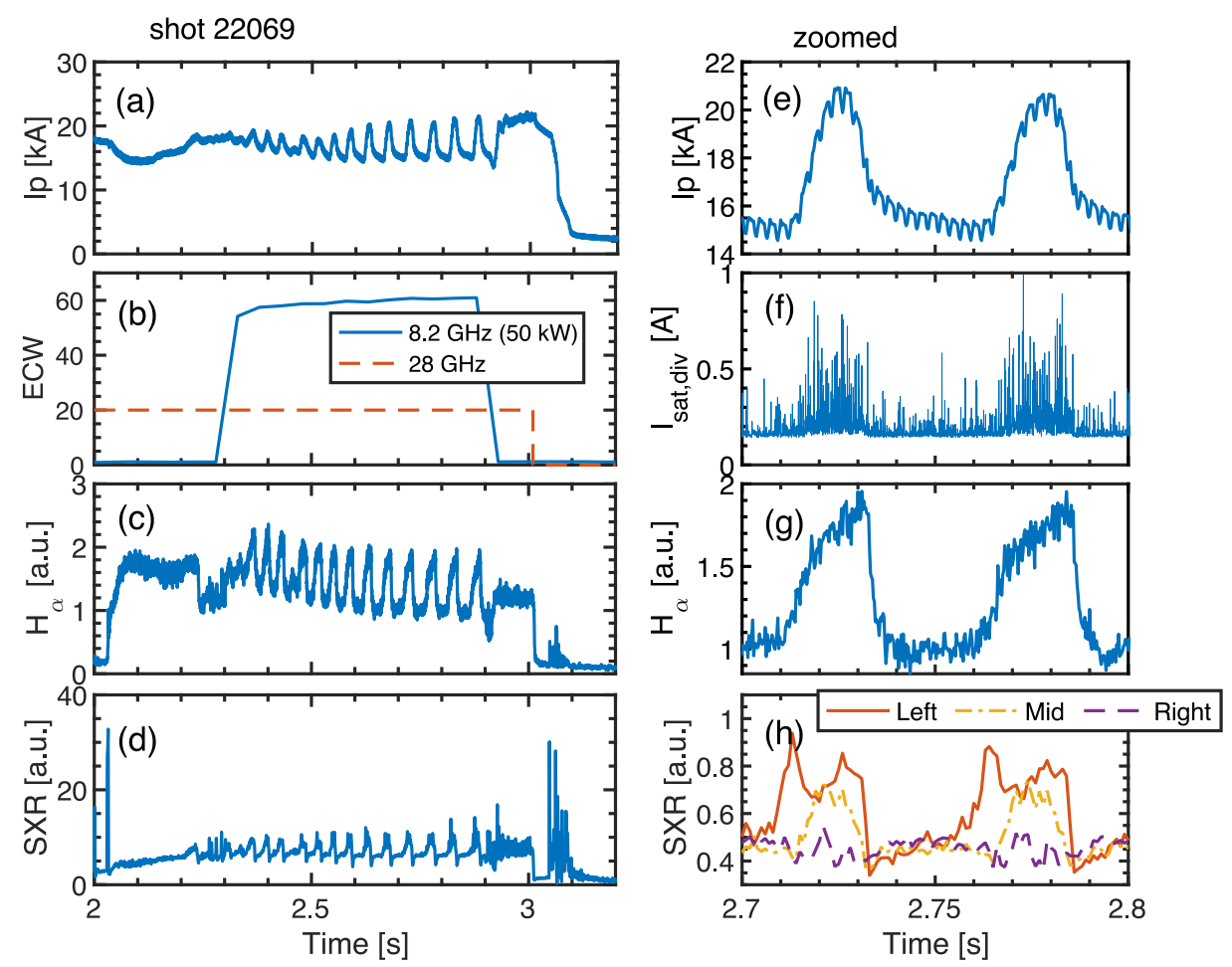

Fig. 9 Waveform of: (a) plasma current, $I_{p}$; (b) 8.2- and 28-GHz ECW heating (the 28-GHz power is much larger than 20 kW); (c) hydrogen Balmer line radiation, $H_{\alpha}$; and (d) SXR intensity. (e-h) Zoomed waveforms of: (e) $I_{p}$, (f) ion saturation current on upper divertor probes, (g) $H_{\alpha}$ and (h) SXR intensity of the three areas shown in Fig. 10.

to the plasma, which efficiently accelerated the electrons driving the plasma current. Visible inspection revealed increases in the carbon and oxygen impurities as a result of the bombardment of slide-away electrons on the plasmafacing wall.

The effect of enhanced impurity ejection as a result of slide-away electron impact was also captured by the SXR imaging system, as shown in Fig. 7 (h). Figures 8 (b) and (c) shows SXR images before and after the emission burst. The time response of impurity events is very fast. The duration is about $1 \sim 2 \mathrm{~ms}$ as detected by SXR images and visible measurement. Unfortunately, the framing rate of $1 \mathrm{kHz}$ is too slow to catch up the details of spatial intensity evolution. In future work, the framing rate can be increased to investigate the behavior of impurity transport using images with high spatial and temporal resolution.

\subsection{Low-frequency plasma oscillation}

The SXR imaging system can also be applied to plasma behavior monitoring. Here, we report on the phenomenon of $\sim 20-\mathrm{Hz}$ low-frequency plasma oscillation and its observation using the SXR imaging system. A typical waveform is shown for shot 22069 in Fig. 9. The plasma 


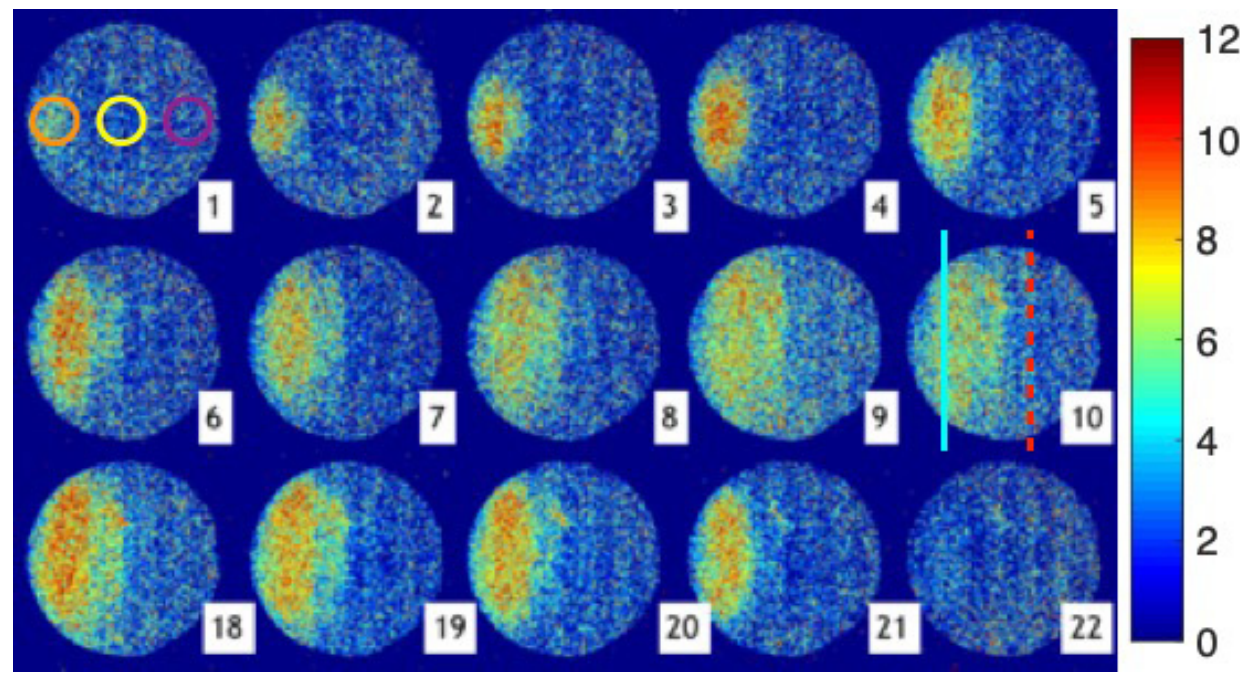

Fig. 10 Images of SXR emissivity (false color) during one cycle of shot 22069.15 out of 50 frames in one cycle are shown. The timing of frame 1 to frame 22 is $2.712 \mathrm{~s}$ to $2.733 \mathrm{~s}$, with a framing rate of $1 \mathrm{kHz}$. Three circular areas chosen to analyze spatial variation are shown in frame $1 ; R_{\text {res } 2}^{28 \mathrm{GHz}}=0.32 \mathrm{~m}$ and $R_{\text {res } 1}^{8.2 \mathrm{GHz}}=0.54 \mathrm{~m}$ are indicated in frame 10 by cyan and red dashed lines, respectively. The range of color map is $[0,12]$.

oscillation occurs from 2.3-2.9 s under the co-application of 8.2 and $28 \mathrm{GHz}$ ECWs. As shown in Fig. 9 (a), the amplitude of $I_{p}$ oscillation increases with time. This lowfrequency oscillation behavior is also seen in the $H_{\alpha}$ and SXR radiation, which are in phase with each other. Although the oscillation is dominant in the plasma core, it can also affect the plasma boundary, as indicated by the ion saturation current signal on the upper divertor probes. Although the cause of this plasma oscillation remains unknown, it is similar to the oscillation phenomenon reported in [21], although only an $8.2 \mathrm{GHz} \mathrm{ECW}$ was applied in that study.

The shape and spatial evolution of a plasma oscillation can be monitored in the imaging mode. Figure 10 shows parts of the plasma oscillation cycle as SXR images. It is seen that the oscillation activity is related to spatial movement in the radial direction. The value of $B_{t}$ is fixed at $0.26 \mathrm{~T}$ during the oscillation and the resonance layers of the 8.2- and 28-GHz ECWs are present within the observation window. The fundamental harmonic resonance layer of the $8.2-\mathrm{GHz} \mathrm{ECW}$ is located at $R_{\text {res } 1}^{8.2 \mathrm{GHz}}=0.54 \mathrm{~m}$ and the second harmonic resonance layer of the $28-\mathrm{GHz} \mathrm{ECW}$ is at $R_{\text {res } 2}^{28 \mathrm{GHz}}=0.32 \mathrm{~m}$. To analyze the spatial variation, the SXR intensities of the left, middle, and right circles in frame 1 of Fig. 10 are compared in Fig. 9(h), from which it is seen that the oscillation affects only the left and middle parts of the view region, corresponding to $R<0.52 \mathrm{~m}$. Estimation of the velocity of radial motion from the images reveals a forward (or outward) velocity of $18 \mathrm{~m} / \mathrm{s}$ and a backward (or inward) velocity of $40 \mathrm{~m} / \mathrm{s}$.

\section{Discussion and Conclusion}

Plasma continuum radiation, which primarily comprises free-free and free-bound bremsstrahlung and recom- bination radiation, is described in [4] using the following formula:

$$
\frac{d P}{d h v} \approx T_{e}^{-1 / 2} \gamma\left(T_{e}, Z\right) Z_{e f f} n_{e}^{2} \times \exp \left\{\frac{-h v}{k T_{e}}\right\},
$$

where $P$ is the radiated X-ray power, $h v$ is the SXR photon energy, $T_{e}, n_{e}$, and $Z_{e f f}$ are the electron temperature, electron density, and average plasma ion charge, respectively, and the factor $\gamma\left(T_{e}, Z\right)$ is the enhancement of the radiated power from recombination radiation, which in QUEST arises predominantly from ionized carbon and oxygen impurities. As seen in Fig. 7 (d), the intensity of SXR radiation from the impurities is about one order higher than that from the hydrogen plasma. Nevertheless, because of recombination radiation it is difficult to calculate $Z_{\text {eff }}$ from the enhanced SXR radiation.

As shown in Fig. 6, $T_{e}$ can be calculated from the energy dependence of the continuum radiation in Eq. (5). Note that, although all of the photons along the line of sight are collected in the photon counting process, the calculated value of $T_{e}$ shown in the figure is the core plasma temperature, not the line-averaged temperature. This is because, at $h v \gg T_{e}$, the outer, cooler regions of the plasma contribute little to the X-ray emission because of the steep exponential fall-off of the spectrum. Thus, at high photon energies one sees only the hottest part of the plasma.

In this study, an SXR imaging system that produces tangential views was installed on QUEST. The system is capable of the rapid imaging of SXR emissions in imaging mode or obtaining SXR spectra and core electron temperatures in photon counting mode. Our planned upgrades to the system include replacing the current "bare" MCP with a CsI-coated MCP for higher detection efficiency (up to the $50 \%$ reported for CsI photocathodes [22]). Another 
enhancement will involve replacing the shutter and Al filter with a vacuum Be window to enable placement of the MCP much closer to the QUEST plasma without incurring arcing from QUEST gas injection. These improvements will extend the viewing area into the edge region, which will enable the calculation of the SXR spectral profile and electron temperature using Abel inversion.

[1] S. Ohdachi et al., Rev. Sci. Instrum. 74(3), 2136 (2003).

[2] J. Mlynar et al., Fusion Eng. Des. 66-68, 905 (2003).

[3] R.D. Gill et al., Nucl. Fusion 40(2), 163 (2000).

[4] L.F. Delgado-Aparicio et al., J. Appl. Phys. 102(7), 073304 (2007).

[5] K. Hanada et al., Plasma Phys. Control. Fusion 32(14), 1289 (1990).

[6] K. Tritz et al., Rev. Sci. Instrum. 83(10), 10E109 (2012).

[7] Y. Liang et al., Rev. Sci. Instrum. 71(10), 3711 (2000).
[8] K. Tritz et al., Rev. Sci. Instrum. 74(3), 2161 (2003).

[9] F. Zhou et al., Rev. Sci. Instrum. 88(7), 073505 (2017).

[10] J.L. Wiza, Nucl. Instrum. Methods 162(1-3), 587 (1979).

[11] K. Hanada et al., Nucl. Fusion 57(12), 126061 (2017).

[12] S. Tashima et al., Nucl. Fusion 54(2), 11 (2014).

[13] M. Ishiguro et al., Phys. Plasmas 19(6), 062508 (2012).

[14] H. Idei et al., Nucl. Fusion 57(12), 126045 (2017).

[15] K. Hanada et al., Plasma Sci. Technol. 13(3), 307 (2011).

[16] K. Hanada et al., Plasma Fusion Res. 5, S1007 (2010).

[17] G.W. Fraser, Nucl. Instrum. Methods Phys. Res. 195(3), 523 (1982).

[18] A. Baciero et al., J. Synchrotron Radiat. 7, 215 (2000).

[19] W. Jin et al., Nucl. Instrum. Methods Phys. Res. A 674, 15 (2012).

[20] L. Delgado-Aparicio et al., Rev. Sci. Instrum. 81(10), 10E303 (2010).

[21] T. Onchi et al., Phys. Plasmas 22(8), 14 (2015).

[22] M.P. Kowalski et al., Appl. Opt. 25(14), 2440 (1986). 